\title{
Assumption-based argumentation for selection and composition of services
}

\author{
Francesca Toni \\ Department of Computing, Imperial College London, UK
}

\begin{abstract}
We present an argumentation-based approach to design and realise agents that can support the selection and composition of services in distributed environments, such as service-oriented architectures and grids. The choice of services (for selection or for composition) is equated to decisions. The agents are equipped with beliefs, in the form of (possibly conflicting) defeasible rules, goals and alternative decisions. Beliefs, goals, decisions may be ranked according to specified preferences. We show how beliefs and preferences can be taken into account to support the decisionmaking process of the agent, in order to achieve its goals. We deal with conflicts and preferences by using assumption-based argumentation, an existing computational-logic-based argumentation framework, that has already been proven to be an effective tool for many applications of argumentation.
\end{abstract}

\section{Introduction}

In recent years, under the influence of the World Wide Web, many standalone software tools have evolved into locally managed but globally accessible applications within heavily distributed environments, such as the grid and serviceoriented architectures. Users are then posed the problem of selecting and/or composing these tools into complex applications, in order to fulfil specified users' needs. The use of agent technology offers a powerful solution to dynamic service composition in distributed settings such as the grid [11]. Different services can be associated with autonomous agents that can identify and negotiate, on behalf of service requestors and providers, implementation plans that take into account the requirements of both sides.

We present an argumentation-based approach to design and realise agents that can support the selection and composition of services in such distributed environments. The choice of services (for selection or for composition) is equated to decisions. The agents are equipped with

- beliefs, in the form of (possibly conflicting) defeasible rules and (defeasible) preferences (e.g. expressing the credibility of beliefs);

- goals (needs/objectives), possibly incompatible and ranked according to users' preferences (e.g. expressing how important they are to the agents); and

- alternative (and thus conflicting) decisions, possibly ranked according to users' preferences (e.g. expressing the cost of decisions). 
Beliefs and preferences need to be taken into account when supporting the decision-making process of the (user) agent, in order to achieve its (most preferred) goals. We deal with conflicts and (qualitative) preferences by using argumentation, and in particular assumption-based argumentation [3, 6, 8-10]. This is a general-purpose framework for argumentation whereby, differently from [7] and work following it, arguments and attack relation are not primitive concepts, but are defined instead: arguments are backward deductions (using sets of rules in an underlying logic) supported by sets of assumptions, and the notion of attack amongst arguments is reduced to that of contrary of assumptions. Assumption-based argumentation has been proven to generalise many forms of non-monotonic reasoning [3] and legal reasoning [14]. It is equipped with correct computational counterparts [8-10] and implementations [12], heavily based upon logic programming.

By using assumption-based argumentation, we basically define argumentative agents capable of solving multi-attribute decision-making problems, where the attributes are seen as the agents' goals, and where information (beliefs) about alternatives (decisions) is not clear-cut, and there are arguments in favour and against attributes taking one or another value. These decisions/goals need to be "weighted" against one another. Moreover, there may be "uncertainty" as to the source/validity of information, or about the current state of the world. Thus the "weight" of arguments may need to take the level of "uncertainty" into account. We represent "weights" and "uncertainty" by using qualitative preferences. We do so inpired by a number of concrete scenarios, borrowed from the ARGUGRID project ${ }^{1}$. These scenarios consider the need for service selection and composition to support business migration and to realise complex earth observation products. These scenarios indicate the need for dealing with possibly conflicting information (epistemic beliefs), decisions linking to goals/objectives, preferences over beliefs, goals, and decisions. In this paper we will show how all these features can be formalised and dealt with by means of assumption-based argumentation.

The paper is organised as follows. In section 2 we describe the motivating scenarios. In section 3 we give some background on assumption-based argumentation. In sections $4,5,6$ we show how to deal with reasoning with beliefs, alternative decisions, and goals (respectively and incrementally) in assumptionbased argumentation. In section 7 we conclude, summarising our contribution, related work and avenues for future research.

\section{Motivating scenarios}

We consider here two example scenarios where service selection and composition are core. These scenarios are central in the ARGUGRID project ${ }^{2}$, that aims at developing a framework supporting service selection and composition over

\footnotetext{
${ }^{1}$ http://www . argugrid .eu

${ }^{2}$ ARGUGRID also considers e-market places scenarios, omitted here for lack of space. Further details on all ARGUGRID scenarios can be found in [19].
} 
the grid and/or service-oriented architectures, with the help of argumentative agents. These scenarios identify the need for a framework capable of handling

- defeasible, conflicting information (beliefs)

- preferences over beliefs

- mutually exclusive decisions for the achievement of goals

- preferences over decisions and goals.

We will see that the framework proposed in this paper will be capable of dealing with this kind of scenarios.

\subsection{Business migration}

Suppose an investor decides to migrate an existing business to a new location, so as to improve profit while fulfilling certain requirements. The investor will typically have (or will be able to get hold of) some information about various alternative locations ("services") and their characteristics.

The choice (selection) of location will typically depend on a number of factors, including ease of accessibility of the location, permits, regulations, and taxes by the national/local governments, local markets and access to global markets, competitors, access to any construction contractors, access to suppliers and materials, availability of additional information or assistance, etc. For example, the investor may require that the proposed location is "easily" accessible, that the tax rate in the country of the chosen location is a maximum of $30 \%$, that there are at least three suppliers of some required materials in the vicinity of the location, and that there are "good" assistance centres for new businesses in the chosen area.

Typically, these factors cannot be assessed with certainty for any given location, as they depend on information that is partial and resulting from sources whose reliability varies. For example, a location may be deemed "easily" accessible because close to a river of sufficient depth for water transportation, according to some source of information. However, if there is no port as yet on that river, the location would be unsuitable for water-transportation, and thus not "easily" accessible. Moreover, according to some other source, the depth of the river may be insufficient at points.

The investor may have some preferences over the requirements and the alternative locations, and these preferences may be conditional. For example, the investor may think that accessibility is a more important factor than availability of materials, as materials can be transported cheaply once the transportation network is set up. Moreover, the investor may prefer a location in a country believed (not) to have signed the Kyoto agreement for climate change control, if (not) environmentally conscious.

Once a location is selected, amongst a number of alternative candidates, several "services" need to be combined, for example a construction contractor and some suppliers. The composition of these services corresponds to a concrete plan of action for the set-up of the business. 


\subsection{Earth observation}

Satellites can be seen as earth observation services, useful in a number of applications (ranging from environmental monitoring, meteorology and map-making) because of their wide area observation capability, the fact that they can provide observations non-intrusively, their capability to provide measurements rapidly and continuosly.

Different earth observation satellites exist, varying, e.g., according to their orbit, according to the kind of instruments they carry on board and, for each instrument, the kind of sensors the instruments are equipped with. The instruments may allow to collect, save, and transmit data about the earth. The sensors may be classified as radar and optical. For example, some satellites (e.g. Meteosat, MGS, Eutelsat) are geostationary (namely apparently stationary wrt a given location on earth), whereas others (e.g. MetOp, Radarsat, JERS_1) are polar (revolving around earth, and passing above both poles at each resolution). Moreover, JERS_1 has radar sensors whereas Meteosat has optical sensors.

Different types of satellites have different characteristics, in terms e.g. of the resolution of images they can record and the frequency with which they can record them, as well as their cost. For example, optical sensors are guaranteed to give higher resolution images than radar sensors, but they are heavily weather-dependent, whereas radar sensors are weather-independent but limited in detecting shapes or surface variations. Also, the RADARSAT-1 satellite would charge $1000 \$$ for emergency programming and $100 \$$ for basic programming.

Given a specific image requirement by a user, in the simplest cases a specific satellite needs to be selected, and, in the more complex cases, a combination of satellites needs to be sought to provide a number of different images. In both cases, the choice needs to take into account the large number of available satellites and their many characteristics, as well as the requirements of the user.

For example, if the image is needed in order to study an oil spill then a single satellite would suffice, as soon as the response time is very quick and the image resolution is high. The choice amongst satellites with radar or optical sensors largely depends on the user's beliefs about the weather. Finally, the choice of the satellite depends on the funds availability of the user and the cost of the satellite. The user may have different preferences concerning its requirements (e.g. an environment-concerned user would definitely give top priority to speed over cost, in the case of an oil spill). Moreover, the user may have (possibly conflicting) beliefs about the time and location of the spill, possibly coming from different sources with different degrees of reliability.

As another example, in case of a fire monitoring scenario, several images possibly from different satellites may be required to control the spreading of a fire over a large region, in conjunction with information about weather conditions. This is an example requiring the combination of services. 


\section{Background: abstract and assumption-based argumentation}

Definition 1. An abstract argumentation framework is a pair (Arg, attacks) where Arg is a finite set, whose elements are referred to as arguments, and attacks $\subseteq$ Arg $\times$ Arg is a binary relation over Arg. Given sets $X, Y \subseteq$ Arg of arguments, $X$ attacks $Y$ iff there exists $x \in X$ and $y \in Y$ such that $(x, y) \in$ attacks.

Given an abstract argumentation framework, several notions of "acceptable" sets of arguments can be defined [7].

Definition 2. A set $X$ of arguments is

- conflict-free iff it does not attack itself;

- admissible iff $X$ is conflict-free and $X$ attacks every set of arguments $Y$ such that $Y$ attacks $X$;

- preferred iff $X$ is maximally admissible;

- sceptically preferred iff $X$ is the intersection of all preferred sets of arguments;

- complete iff $X$ is admissible and $X$ contains all arguments $x$ such that $X$ attacks all attacks against $x$;

- grounded iff $X$ is minimally complete;

- ideal iff $X$ is admissible and it is contained in every preferred set of arguments.

The last notion was not in the original [7], but has been proposed recently [9, 10] as an alternative, less sceptical semantics than the grounded semantics.

The abstract view of argumentation does not deal with the problem of actually finding arguments and attacks amongst them. Typically, arguments are built by connecting rules in the belief set of the proponent of arguments, and attacks arise from conflicts amongst such arguments. In assumption-based argumentation, arguments are (implicitly meant to be) obtained by reasoning backwards with a given set of inference rules (the belief set), from conclusions to premises that are assumptions, and attacks are defined in terms of a notion of "contrary" of assumptions. Belief set and backward reasoning are defined in terms of a deductive system:

Definition 3. $A$ deductive system is a pair $(\mathcal{L}, \mathcal{R})$ where

- $\mathcal{L}$ is a formal language consisting of countably many sentences, and

$-\mathcal{R}$ is a countable set of inference rules of the form

$$
\frac{x_{1}, \ldots, x_{n}}{x}
$$

where $x \in \mathcal{L}$ is called the conclusion and $x_{1}, \ldots, x_{n} \in \mathcal{L}$ are called the premises of the inference rule, and $n \geq 0$. 
If $n=0$, then the inference rule represents an axiom. Note that a deductive system does not distinguish between domain-independent axioms/rules, which belong to the specification of the logic, and domain-dependent axioms/rules, which represent a background theory. For notational convenience, throughout the paper we write $x \leftarrow x_{1}, \ldots, x_{n}$ instead of $\frac{x_{1}, \ldots, x_{n}}{x}$ and $x$ instead of $x \leftarrow$.

Definition 4. Given a deductive system $(\mathcal{L}, \mathcal{R})$ and a selection function ${ }^{3} f$, $a$ (backward) deduction of a conclusion $x$ based on (or supported by) a set of premises $P$ is a sequence of multi-sets $S_{1}, \ldots, S_{m}$, where $S_{1}=\{x\}, S_{m}=P$, and for every $1 \leq i<m$, where $y$ is the sentence occurrence in $S_{i}$ selected by $f$ :

1. If $y$ is not in $P$ then $S_{i+1}=S_{i}-\{y\} \cup S$ for some inference rule of the form $y \leftarrow S \in \mathcal{R} .{ }^{4}$

2. If $y$ is in $P$ then $S_{i+1}=S_{i}$.

Each $S_{i}$ is referred to as a step in the deduction.

In the remainder of this paper we will use the following notation: $P \vdash c$ will stand for "there exists a deduction of $c$ supported by $P$ ". This notation is simplistic as it does not allow to distinguish different deductions to the same conclusions and supported by the same premises, but it is a useful shorthand when the steps in the deduction are not of interest.

Deductions are the basis for the construction of arguments in assumptionbased argumentation, but to obtain an argument from a backward deduction we restrict the premises to ones that are assumptions. Moreover, to specify when one argument attacks another, we need to specify contraries of assumptions.

Definition 5. An assumption-based argumentation framework is a tuple $\langle\mathcal{L}, \mathcal{R}, \mathcal{A},-\rangle$ where

- $(\mathcal{L}, \mathcal{R})$ is a deductive system.

$-\mathcal{A} \subseteq \mathcal{L}, \mathcal{A} \neq\{\} . \mathcal{A}$ is referred to as the set of assumptions.

- If $\bar{x} \in \mathcal{A}$, then there is no inference rule of the form $x \leftarrow x_{1}, \ldots, x_{n} \in \mathcal{R}$.

- ${ }^{-}$is a (total) mapping from $\mathcal{A}$ into $\mathcal{L} . \bar{x}$ is referred to as the contrary of $x$.

Note that assumption-based frameworks are still abstract, in the sense that in order to be deployed they need to be instantiated. Several instances have been studied already $[3,14]$. In this paper we study some additional instances, for epistemic and practical reasoning. Note that, by the third bullet, following [8] we restrict ourselves to flat frameworks [3], whose assumptions do not occur as

\footnotetext{
${ }^{3}$ A selection function is any function from sets of elements to elements. The definition of backward deduction relies upon some chosen selection function. However, note that if a backward deduction for a conclusion exists for some selection function, then a backward deduction for that conclusion will exist for any other selection function. This result follows from the analogous result for SLD-resolution for Horn clauses.

${ }^{4}$ The same symbols are used for multi-set membership, union, intersection and subtraction as for ordinary sets.
} 
conclusions of inference rules. Flat frameworks are restricted but still interesting and general, as, for example, they admit default logic and logic programming as concrete instances [3], as well as all the instances we will consider in this paper.

In the assumption-based approach to argumentation, arguments are deductions to conclusions, based solely upon assumptions, and the attack relationship between arguments depends solely on the contrary of assumptions.

Definition 6. $A$ set of assumptions $A$ attacks a set of assumptions $B$ iff there exists an assumption $x \in B$ and a deduction $A^{\prime} \vdash \bar{x}$ such that $A^{\prime} \subseteq A$ : if this is the case, we say that $A$ attacks $B$ on $x$.

This notion of attack between sets of assumptions implicitly gives a notion of attack between arguments supported by sets of assumptions: the attacking argument needs to have as conclusion the contrary of an assumption in the support of the attacked argument.

Within assumption-based argumentation, implicitly, a set of assumptions stands for the set of all arguments whose premises are contained in the given set of assumptions. Thus, the computation of "acceptable" sets of arguments amounts to computing "acceptable" sets of assumptions:

Definition 7. A set $X$ of assumptions is

- conflict-free iff $X$ does not attack itself;

- admissible iff $X$ conflict-free and $X$ attacks every set of assumptions $Y$ that attacks $X$;

- preferred iff it is maximally admissible;

- sceptically preferred iff $X$ is the intersection of all preferred sets of assumptions;

- complete iff it is admissible and contains all assumptions $x$ such that $X$ attacks all attacks against $\{x\}$;

- grounded iff it is minimally complete;

- ideal iff $X$ is admissible and it is contained in every preferred set of assumptions.

\section{Reasoning about beliefs}

Reasoning about beliefs may be performed within a framework consisting of defeasible and strict rules and facts [17], some of which may express preferences over the application of rules and the use of facts (thus some of these preferences may be themselves defeasible). The use of rules to represent preferences rather than fixed partial orders is advocated by many, e.g. $[4,18]$, driven by the requirements of applications, for example in a legal domain. We define here frameworks for reasoning about beliefs (referred to as epistemic frameworks) based upon defeasible rules and preferences, ignoring for simplicity strict rules. ${ }^{5}$ We start by giving some preliminary notions.

\footnotetext{
${ }^{5}$ Strict rules require special attention to guarantee "closedness" and "consistency" of epistemic reasoning [5]. For a treatment of strict rules in epistemic frameworks built upon assumption-based argumentation see [20].
} 


\section{Definition 8.}

- A language $\mathcal{L}_{a g}$ is a set of ground literals, which can be atoms a or negations of atoms $\neg$. We will refer to these literals as basic literals.

- A naming $\mathcal{N}$ is a bijective function associating a distinguished name $\mathcal{N}(x)$ to any element $x$ in a given domain $X$. For any given $X$, we will refer to the set of all such names as $\mathcal{N}(X)$.

- A preference literal (wrt $X$ and $\mathcal{N}$ ) is of the form $n_{1} \succ n_{2}$ where $n_{1}, n_{2}$ are (different) names in $\mathcal{N}(X)$.

- A literal is either a basic or a preference literal.

Intuitively, $\mathcal{L}_{a g}$ is the language underlying the agent's reasoning problem, in the chosen domain. When $X$ is a given set of rules over $\mathcal{L}_{a g}, n_{1} \succ n_{2}$ stands for "the rule named $n_{1}$ is preferred to the rule named $n_{2}$ ". In the remainder of this paper, given a basic literal $l$, with an abuse of notation, $\neg l$ will stand for the complement of $l$, namely $\neg l$ if $l$ is an atom, and $a$ if $l$ is a negative literal $\neg a$. Moreover, given a preference literal $l$ of the form $n_{1} \succ n_{2}, \neg l$ will stand for $n_{2} \succ n_{1}$.

Definition 9. Given a language $\mathcal{L}_{\text {ag }}$ and a naming $\mathcal{N}$ :

- $A$ basic rule (wrt $\mathcal{L}_{a g}$ ) is of the form $P \rightarrow c$ where $P=l_{1}, \ldots, l_{n}$ and $c, l_{1}, \ldots, l_{n}$ are basic literals in $\mathcal{L}_{a g}$ and $n \geq 0$.

- $A$ preference rule (wrt some given $X$ and $\mathcal{N}$ ) is of the form $P \rightarrow c$ where $c$ is a preference literal (wrt $X$ and $\mathcal{N}$ ), $P=l_{1}, \ldots, l_{n}$ and $l_{1}, \ldots, l_{n}$ are basic literals in $\mathcal{L}_{a g}$ or preference literals (wrt $X$ and $\mathcal{N}$ ), and $n \geq 0$.

- A (defeasible) rule is either a basic rule or a preference rule.

Given a rule $P \rightarrow c, c$ is referred to as the conclusion and $P$ as the premises. When $n=0$ the rule may be referred to as a fact.

Definition 10. Let $\mathcal{L}_{a g}$ be a language and $\mathcal{N}$ be a naming. An epistemic framework $\epsilon$ is a set $E$ of defeasible rules that can be partitioned into sets $E_{1}, \ldots, E_{d}$, $d \geq 0$, such that

- $E_{1}$ is a set of basic rules (wrt $\mathcal{L}_{a g}$ );

- for each $i \geq 1, E_{i}$ is a set of preference rules wrt $X=\cup_{j=1, \ldots, i-1} E_{j}$ and $\mathcal{N}$.

Intuitively, defeasible rules may or may not be chosen by a rational reasoner, depending on the emergence of conflicts. A rational reasoner needs to avoid these conflicts in its chosen "reasoning lines". Conflicts in epistemic frameworks arise from "deriving" complementary conclusions from sets of chosen (defeasible) rules, either of the form $a$ and $\neg a$ or of the form $n_{1} \succ n_{2}$ and $n_{2} \succ n_{1}$. The semantics of epistemic frameworks needs to resolve these conflicts. In the remainder of this section, we will show how to provide this semantics for epistemic frameworks by means of assumption-based argumentation, by first considering frameworks without preference rules.

Below, we will assume given an epistemic framework $\epsilon=E$ wrt a language $\mathcal{L}_{a g}$ and a naming $\mathcal{N}$. When we restrict attention to epistemic frameworks without preference rules, $\mathcal{L}_{a g}$ will consist solely of basic literals (and $\mathcal{N}$ will be ignored). 
Definition 11. The assumption-based framework corresponding to an epistemic framework without preferences $\epsilon=E$ is $\left\langle\mathcal{L}_{\epsilon}, \mathcal{R}_{\epsilon}, \mathcal{A}_{\epsilon},-\right\rangle$ whereby

- $\mathcal{A}_{\epsilon}$ is a set of literals not already in $\mathcal{L}_{a g}$ such that there exists a bijective mapping a from rules in $E$ into $\mathcal{A}_{\epsilon}$;

$-\mathcal{L}_{\epsilon}=\mathcal{L}_{a g} \cup \mathcal{A}_{\epsilon}$

$-\underline{\mathcal{R}_{\epsilon}=\{c \leftarrow P, \alpha(P \rightarrow c) \mid P \rightarrow c \in E\}}$

$-\overline{\alpha(P \rightarrow c)}=\neg c$.

Intuitively, any assumption in $\mathcal{A}_{\epsilon}$ correspond to the applicability of the corresponding rule, which is opposed by the complement of the conclusion of that rule being "derivable": this is expressed by the definition of contrary.

Example 1. Consider $E=\{q ; q \rightarrow p ; r ; r \rightarrow \neg p\}$. In the context of the business migration scenario of section $2, p$ may represent that some given country of interest has signed the Tokyo protocol. There is evidence from some source $q$ that this is so $(q \rightarrow p)$, and evidence from some other source $r$ that this is not so $(r \rightarrow \neg p)$. The sources ( $q$ and $r$ ) are defeasible, and so are the reports claiming that these sources provided support for $p$ and $\neg p$ (that the given country signed/did not sign the Tokyo protocol respectively).

Given this $E$, we can choose $\mathcal{A}_{\epsilon}=\left\{a_{1}, a_{2}, a_{3}, a_{4}\right\}{ }^{6}$, and then $\mathcal{R}_{\epsilon}=\{q \leftarrow$ $\left.a_{1} ; p \leftarrow q, a_{2} ; r \leftarrow a_{3} ; \neg p \leftarrow r, a_{4}\right\}$, and $\overline{a_{1}}=\neg q, \overline{a_{2}}=\neg p, \overline{a_{3}}=\neg r$, and $\overline{a_{4}}=p$.

By virtue of the formulation in definition 11, any notion of acceptable set of assumptions may be adopted to provide a semantics to $E$. For instance, in example $1,\left\{a_{1}, a_{2}\right\}$ is an admissible set of assumptions with $\{q, p\}$ the corresponding "output" beliefs, and $\left\{a_{1}, a_{3}\right\}$ is the grounded set of assumptions with $\{q, r\}$ the corresponding "output" beliefs.

Let us now consider epistemic frameworks with preference rules. We will use the following notation: given a set of rule $X$, a literal $L$ is defined in $X$ if a rule in $X$ has $L$ or $\neg L$ as its conclusion.

Definition 12. The assumption-based framework corresponding to an epistemic framework with preferences $\epsilon=E$ is $\left\langle\mathcal{L}_{\epsilon}, \mathcal{R}_{\epsilon}, \mathcal{A}_{\epsilon},-\right\rangle$ whereby

- $\mathcal{A}_{\epsilon}$ is a set of literals not already in $\mathcal{L}_{a g}$ such that there exists a bijective mapping a from rules in $E$ into $\mathcal{A}_{\epsilon}$;

- $\mathcal{L}_{\epsilon}=\mathcal{L}_{a g} \cup \mathcal{A}_{\epsilon} \cup \mathcal{B}_{\epsilon} \cup \mathcal{C}_{\epsilon}$ where $\mathcal{B}_{\epsilon}$ and $\mathcal{C}_{\epsilon}$ are distinct sets of literals not already in $\mathcal{L}_{a g} \cup \mathcal{A}_{\epsilon}$ such that

- there exists a bijective mapping $\beta$ from rules in $E$ into $\mathcal{B}_{\epsilon}$;

- there exists a bijective mapping $\chi$ from assumptions in $\mathcal{A}_{\epsilon}$ into $\mathcal{C}_{\epsilon}$;

${ }^{6}$ Note that the specification of $\mathcal{R}_{\epsilon}$ depends on the choice of $\mathcal{A}_{\epsilon}$, and for each different choice of $\mathcal{A}_{\epsilon}$ a different $\mathcal{R}_{\epsilon}$ needs to be given. However, all alternative choices are isomorphic. 


$$
\begin{aligned}
-\mathcal{R}_{\epsilon}= & \{c \leftarrow \beta(P \rightarrow c) \mid P \rightarrow c \in E\} \cup \\
& \{\beta(P \rightarrow c) \leftarrow P, \alpha(P \rightarrow c) \mid P \rightarrow c \in E\} \cup \\
& \left\{\chi(a) \leftarrow n^{\prime} \succ n, \beta\left(P^{\prime} \rightarrow \neg c\right) \mid a=\alpha(P \rightarrow c), P \rightarrow c \in E, P^{\prime} \rightarrow \neg c \in E,\right. \\
& n=\mathcal{N}(P \rightarrow c), n^{\prime}=\mathcal{N}\left(P^{\prime} \rightarrow \neg c\right), \\
& \left.n^{\prime} \succ n \text { is defined in } E\right\} \cup \\
& \left\{\chi(a) \leftarrow \beta\left(P^{\prime} \rightarrow \neg c\right) \mid a=\alpha(P \rightarrow c), P \rightarrow c \in E, P^{\prime} \rightarrow \neg \in E,\right. \\
& \left.\mathcal{N}(P \rightarrow c) \succ \mathcal{N}\left(P^{\prime} \rightarrow \neg c\right) \text { is not defined in } E\right\} ; \\
-\bar{a}= & \chi(a) .
\end{aligned}
$$

Intuitively, assumptions in $\mathcal{A}_{\epsilon}$, as in the case of no preference rules, correspond to the applicability of the corresponding rules, sentences in $\mathcal{B}_{\epsilon}$ correspond to the actual application of the corresponding rules, and sentences in $\mathcal{C}_{\epsilon}$ correspond to objecting to the application of a rule, by a rule with higher preference and conflicting conclusion being "derivable" (if any) or simply by a rule with conficting conclusion (if no preference is defined): this is expressed by the definition of contrary.

Example 2. Consider $E=\left\{q \rightarrow p ; q ; \neg p ; \neg q ; r \rightarrow n_{1} \succ n_{3} ; r ; \neg r\right\}$, where $n_{1}=$ $\mathcal{N}(q \rightarrow p)$ and $n_{3}=\mathcal{N}(\neg p)$. Here, similarly to example $1, p$ may represent, in the context of the business migration scenario of section 2, that some given country of interest has signed the Tokyo protocol, with $q \rightarrow p$ representing that there is evidence that some source $q$ claims that this is indeed so. The fact that the source $q$ is reliably making this claim is debatable (both $q$ and $\neg q$ are rules). The rule $\neg p$ may represent a defeasible belief that the country of interest has not signed the Tokyo protocol, and the preference rule $r \rightarrow n_{1} \succ n_{3}$ may represent that there is evidence from some source $r$ supporting that the rule leading to conclude $p$ is stronger (more reliable than) the rule concluding $\neg p$.

Given this $E, \mathcal{A}_{\epsilon}$ may be $\left\{a_{1}, a_{2}, a_{3}, a_{4}, a_{5}, a_{6}, a_{7}\right\}, \mathcal{R}_{\epsilon}$ is

$$
\begin{aligned}
& \left\{p \leftarrow b_{1} ; \quad b_{1} \leftarrow q, a_{1} ; \quad c_{1} \leftarrow n_{3} \succ n_{1}, b_{3} ;\right. \\
& q \leftarrow b_{2} ; b_{2} \leftarrow a_{2} ; \quad c_{2} \leftarrow b_{4} ; \\
& \neg p \leftarrow b_{3} ; \quad b_{3} \leftarrow a_{3} ; \quad c_{3} \leftarrow n_{1} \succ n_{3}, b_{1} ; \\
& \neg q \leftarrow b_{4} ; b_{4} \leftarrow a_{4} ; \quad c_{4} \leftarrow b_{2} ; \\
& n_{1} \succ n_{3} \leftarrow b_{5} ; \quad b_{5} \leftarrow r, a_{5} ; \\
& r \leftarrow b_{6} ; b_{6} \leftarrow a_{6} ; c_{6} \leftarrow b_{7} ; \\
& \left.\neg r \leftarrow b_{7} ; b_{7} \leftarrow a_{7} ; c_{7} \leftarrow b_{6}\right\}
\end{aligned}
$$

and $\overline{a_{i}}=c_{i}$, for $i=1, \ldots, 7$.

By virtue of the formulation in definition 12, any notion of "acceptable" set of assumptions may be adopted to provide a semantics for $E$. For instance, in example $2,\left\{a_{1}, a_{2}\right\}$ is an admissible set of assumptions, with "output" beliefs $\{p, q\}$. Indeed, this set of assumptions is conflict-free (there is no backward deduction from any of its subsets supporting any of $c_{1}, c_{2}$ ). Moreover, it attacks (by means of a deduction supporting $c_{4}$ ) the set of assumptions $\left\{a_{4}\right\}$ that attacks it (by means of a deduction supporting $c_{2}$ ). Note that the assumption $a_{1}$ is not 
attacked by any set of assumptions, as there is no deduction supporting $c_{1}$. The set of assumptions $\left\{a_{3}, a_{4}\right\}$ is also admissible, as it is conflict-free and it attacks all attacks against it: it is attacked by

- $\left\{a_{1}, a_{2}, a_{5}, a_{6}\right\}$ (supporting a deduction for $c_{3}$ ), counter-attacked by $\left\{a_{4}\right\}$,

- $\left\{a_{2}\right\}$ (supporting a deduction for $c_{4}$ ), also counter-attacked by $\left\{a_{4}\right\}$.

\section{$5 \quad$ Reasoning about decisions}

Definition 13. Given $\mathcal{L}_{a g}$, a practical framework is a tuple $\langle\epsilon, \mathcal{D}, \mathcal{G}, D\rangle$ where

$-\epsilon$ is an epistemic framework wrt $\mathcal{L}_{a g}$;

$-\mathcal{D} \subseteq \mathcal{L}_{a g}$ is the set of potential decisions such that none of its elements occurs in the conclusion of any rule in $\epsilon$;

$-\mathcal{G} \subseteq \mathcal{L}_{a g}$ is the set of goals;

$-D$ is a set of preference rules wrt $\mathcal{D}$ and some naming (and wrt $\mathcal{L}_{\text {ag }}$ ).

In the remainder of the paper, we will assume that the naming is the identity function, associating $d$ to each decision $d \in \mathcal{D}$.

Intuitively, the agent's task is to choose amongst its potential decisions in $\mathcal{D}$ so that its goals in $\mathcal{G}$ are achieved. These decisions are intended to be mutually exclusive alternatives, that can be understood as possible plans of actions. The goals are objectives/constraints of the agent, namely features that the agent would like the decisions to exhibit.

The agent's preferences amongst these decisions are represented within $D$. These preferences may be expressing a partial order, when $D$ only consists of facts (and special care is taken so that antisymmetry holds).More generally, $D$ is a set of preference rules, e.g. $P \rightarrow d_{1} \succ d_{2}$, expressing that a decision $d_{1}$ is preferred to another decision $d_{2}$ under certain circumstances $P$. These circumstances are represented by means of literals in $\mathcal{L}_{a g}$, and will typically be beliefs to be evaluated wrt $\epsilon$.

From now on we will assume given $\langle\epsilon, \mathcal{D}, \mathcal{G}, D\rangle$ wrt $\mathcal{L}_{a g}$. We will first consider the case where $D=\{\}$, and then the general case.

Frameworks for practical reasoning can be modelled naturally within generalised assumption-based frameworks ${ }^{7}$, whereby contrary is a (total) mapping from assumptions into sets of sentences in $\mathcal{L}$ (rather than individual sentences in $\mathcal{L}$ ). Given such a generalised framework, the notion of attack between sets of assumptions is modified as follows

- a set of assumptions $X$ attacks a set of assumptions $Y$ iff there exists an assumption $x \in Y$, a sentence $y \in \bar{x}$ and an argument $X^{\prime} \vdash y$ such that $X^{\prime} \subseteq X$.

\footnotetext{
${ }^{7}$ Note that this generalisation is strictly speaking not necessary, and practical frameworks could be expressed also within the orginal form of assumption-based frameworks, by introducing new sentences and rules in the underlying deductive system, but somewhat less naturally.
} 
Definition 14. The (generalised) assumption-based framework corresponding to $\pi=\langle\epsilon, \mathcal{D}, \mathcal{G},\{\}\rangle$ is $\left\langle\mathcal{L}_{\pi}, \mathcal{R}_{\pi}, \mathcal{A}_{\pi},-\right\rangle$ whereby, given that $\left\langle\mathcal{L}_{\epsilon}, \mathcal{R}_{\epsilon}, \mathcal{A}_{\epsilon},-\right\rangle$ is the assumption-based framework corresponding to $\epsilon$,

$-\mathcal{A}_{\pi}=\mathcal{A}_{\epsilon} \cup \mathcal{D}$

$-\mathcal{L}_{\pi}=\mathcal{L}_{\epsilon}$

$-\mathcal{R}_{\pi}=\mathcal{R}_{\epsilon}$;

- if $x \in \mathcal{A}_{\epsilon}$, then $\bar{x}=\{y\}$ where $y$ is the contrary of $x$ in $\left\langle\mathcal{L}_{\epsilon}, \mathcal{R}_{\epsilon}, \mathcal{A}_{\epsilon},-\right\rangle$; if $x \in \mathcal{D}$, then $\bar{x}=\mathcal{D}-\{x\}$.

Intuitively, the decisions correspond to new assumptions, reflecting the "abductive" nature of decision-making. The mutual exclusion amongst decisions is achieved by setting the contrary of decisions to all other decisions.

Below, whenever $\bar{x}=\{y\}$, for $x, y \in \mathcal{L}_{a g}$, we will write simply $\bar{x}=y$.

Example 3. Consider $\pi=\langle\epsilon, \mathcal{D}, \mathcal{G},\{\}\rangle$ where $\epsilon=E=\left\{p ; d_{1}, p \rightarrow q ; d_{2} \rightarrow s ; s \rightarrow\right.$ $\left.t ; t \rightarrow \neg p ; d_{1} \rightarrow \neg p\right\}$ and $\mathcal{D}=\left\{d_{1}, d_{2}\right\}$. Here, again in the context of the business migration scenario of section $2, d_{1}$ and $d_{2}$ may represent two alternative locations for the migration of a business, each having benefits $\left(s\right.$ for $d_{2}, \neg p$ for $d_{1}$ and, if the belief $p$ is held, also $q$ for $d_{1}$; each of these benefits may have repercussions). Then, in $\left\langle\mathcal{L}_{\pi}, \mathcal{R}_{\pi}, \mathcal{A}_{\pi},{ }^{-}\right\rangle, \mathcal{R}_{\pi}$ is ${ }^{8}$ :

$$
p \leftarrow a_{1} ; \quad q \leftarrow p, d_{1}, a_{2} ; \quad s \leftarrow d_{2}, a_{3} ; \quad t \leftarrow s, a_{4} ; \quad \neg p \leftarrow t, a_{5} ; \quad \neg p \leftarrow d_{1}, a_{6}
$$

where $\mathcal{A}_{\pi}=\left\{a_{1}, \ldots, a_{6}, d_{1}, d_{2}\right\}$ and $\overline{a_{1}}=\neg p, \overline{a_{2}}=\neg q, \overline{a_{3}}=\neg s, \overline{a_{4}}=\neg t$, $\overline{a_{5}}=\overline{a_{6}}=p, \overline{d_{1}}=d_{2}, \overline{d_{2}}=d_{1}$.

By virtue of this formulation, any notion of "acceptable" set of assumptions may be adopted to provide a semantics to $\langle\epsilon, \mathcal{D}, \mathcal{G}, D\rangle$. For instance, in example 3 , $\left\{a_{1}, a_{2}, d_{1}\right\}$ and $\left\{a_{3}, a_{4}, a_{5}, d_{2}\right\}$ are both admissible sets of assumptions, with corresponding "outputs" $\{p, q\}$ and $\{s, t, \neg p\}$, respectively.

Amongst all acceptable sets of arguments, we want to consider solely those having the goals in $\mathcal{G}$ in their "output".

Definition 15. Given $\left\langle\mathcal{L}_{\pi}, \mathcal{R}_{\pi}, \mathcal{A}_{\pi},-\right\rangle$, an "acceptable" set of assumptions $\Delta$ wrt $\left\langle\mathcal{L}_{\pi}, \mathcal{R}_{\pi}, \mathcal{A}_{\pi}, \longrightarrow\right\rangle$ is desired iff $\mathcal{G} \subseteq O(\Delta)$, where $O(\Delta)=\left\{x \in \mathcal{L}_{\pi} \mid \Delta^{\prime} \vdash_{\pi}\right.$ $\left.x, \Delta^{\prime} \subseteq \Delta\right\}$, with $\Delta^{\prime} \vdash_{\pi} x$ standing for "there exists a deduction of $x$ supported by $\Delta^{\prime} "$ wrt $\left\langle\mathcal{L}_{\pi}, \mathcal{R}_{\pi}, \mathcal{A}_{\pi},-\right\rangle$.

Thus, practical reasoning may be realised within assumption-based argumentation by identifying acceptable sets of assumptions that contain a support for the desired goals. For instance, given $\mathcal{G}=\{\neg p\}$ in example $3,\left\{a_{3}, a_{4}, a_{5}, d_{2}\right\}$ is desired admissible, whereas $\left\{a_{1}, a_{2}, d_{1}\right\}$ is not.

\footnotetext{
${ }^{8}$ We adopt here the simpler translation given in section 4 , as there are no preference rules in $\epsilon$. Note that the specification of $\mathcal{R}_{\pi}$ depends on the choice of $\mathcal{A}_{\pi}$, and for each different choice of $\mathcal{A}_{\pi}$ a different $\mathcal{R}_{\pi}$ needs to be given. However, all alternative choices are isomorphic.
} 
Note that $\mathcal{G}$ may contain positive and negative literals. Thus, implicitly, $\mathcal{G}$ specifies properties that need to be fulfilled (the positive literals) and properties that need to be avoided (the negative literals).

Let us consider now the general case of practical frameworks $\langle\epsilon, \mathcal{D}, \mathcal{G}, D\rangle$ with any, possibly non-empty $D$. The translation of definition 14 can be generalised so that rules in $D$ are treated in a similar manner as preference rules in $\epsilon$. This is illustrated by the following example.

Example 4. Consider $\pi=\langle\epsilon, \mathcal{D}, \mathcal{G}, D\rangle$ where $\epsilon$ and $\mathcal{D}$ are as in example 3 , and $D=\left\{u \rightarrow d_{1} \succ d_{2} ; v \rightarrow d_{2} \succ d_{1}\right\}$, where $u, v$ are new atoms of $\mathcal{L}_{a g}$. Then, in $\left\langle\mathcal{L}_{\pi}, \mathcal{R}_{\pi}, \mathcal{A}_{\pi},-\right\rangle, \mathcal{R}_{\pi}$ is

$$
\begin{aligned}
& p \leftarrow a_{1} ; \quad q \leftarrow p, d_{1}, a_{2} ; \quad s \leftarrow d_{2}, a_{3} ; \quad t \leftarrow s, a_{4} ; \quad \neg p \leftarrow t, a_{5} ; \quad \neg p \leftarrow d_{1}, a_{6} ; \\
& d_{1} \succ d_{2} \leftarrow b_{7} ; \quad b_{7} \leftarrow u, a_{7} ; \quad c_{7} \leftarrow b_{8} ; \\
& d_{2} \succ d_{1} \leftarrow b_{8} \quad b_{8} \leftarrow v, a_{8} ; \quad c_{8} \leftarrow b_{7} ; \\
& e_{1} \leftarrow d_{2} \succ d_{1} ; \quad e_{1} \leftarrow d_{2} ; \\
& e_{2} \leftarrow d_{1} \succ d_{2} ; \quad e_{2} \leftarrow d_{1}
\end{aligned}
$$

where $\mathcal{A}_{\pi}=\left\{a_{1}, \ldots, a_{8}, d_{1}, d_{2}\right\}$ and $\overline{a_{1}}=\neg p, \overline{a_{2}}=\neg q, \overline{a_{3}}=\neg s, \overline{a_{4}}=\neg t$, $\overline{a_{5}}=\overline{a_{6}}=p, \overline{a_{7}}=c_{7}, \overline{a_{8}}=c_{8}, \overline{d_{1}}=e_{2}, \overline{d_{2}}=e_{1}$. The translation introduces new literals $\left(e_{1}, e_{2}\right)$ for the contraries of decisions and new definitions for these contraries (last two lines in the description of $\mathcal{R}_{\pi}$ ) preventing decisions being made if more preferred decisions can be made too, and in any case preventing incompatible decisions. In the example, if $u$ is an additional fact in $\epsilon$, then $d_{1}$ is the only decision possible to achieve the goal $\neg p$ according to any argumentation semantics for $\left\langle\mathcal{L}_{\pi}, \mathcal{R}_{\pi}, \mathcal{A}_{\pi},-\right\rangle$.

Due to lack of space, we omit here the formal translation into assumption-based argumentation for $\langle\epsilon, \mathcal{D}, \mathcal{G}, D\rangle$ with a non-empty $D$.

So far we have assumed that decisions are all in a single pool $\mathcal{D}$, but in general there could be sets of sets of potential decisions, and composite decisions resulting from choosing one element for each set. For example, for service composition, two services may be needed, and for each of these services a number of possible choices may be available. This can be accommodated in a straightforward manner, as follows:

- $\mathcal{D}$ is partitioned into subsets $\mathcal{D}_{1}, \ldots, \mathcal{D}_{n}, n \geq 1$

- in $\left\langle\mathcal{L}_{\pi}, \mathcal{R}_{\pi}, \mathcal{A}_{\pi},-\right\rangle$, all elements of $\mathcal{D}_{i}$ are assumptions in $\mathcal{A}_{\pi}$;

- in $\left\langle\mathcal{L}_{\pi}, \mathcal{R}_{\pi}, \mathcal{A}_{\pi},-\right\rangle$, for each $i$, for each $x \in \mathcal{D}_{i}$, the contrary of $x$ is set to $\mathcal{D}_{i}-\{x\}$

- the preference rules in $D$ may be given only for decisions in the same element of the partition of $\mathcal{D}$.

For example, in the context of the business migration scenario of section 2, there may $\mathcal{D}_{1}$ and $\mathcal{D}_{2}$, the first representing a number of alternatives for building constructors, the second a number of alternatives for suppliers of materials. We omit further details due to lack of space. 


\section{Reasoning about goals}

So far we have assumed that all goals in $\mathcal{G}$ that the decisions aim at achieving are equally important. Also, in some cases no desired "acceptable" decision (set of assumptions) may exist, e.g. if the goals are incompatible. This may happen in example 3 , for instance, given $\mathcal{G}=\{\neg p, q\}$. The use of stratification or, more generally, user-defined preferences over $\mathcal{G}$ will help in general with identifying desired "acceptable" sets. For example, if $\mathcal{G}=\{\neg p, q\}$ and $\neg p \succ q$ (namely the goal $\neg p$ is more preferred than the goal $q$ ), then $\left\{a_{3}, a_{4}, a_{5}, d_{2}\right\}$ is desired admissible, if instead $q \succ \neg p$ (namely the goal $q$ is more preferred than the goal $\neg p$ ), then $\left\{a_{1}, a_{2}, d_{1}\right\}$ is desired admissible.

Preferences may be seen as providing several layers of importance for goals (from "must have" to various degrees of "wish to have"). In the case of preferences providing a fixed partial order (given by means of facts, with appropriate antysymmetry), the preferences provide a partition/stratification whereby the highest layer correspond to properties whose achievement/avoidance is a must for the agent, and the remaining layers correspond to properties whose achievement/avoidance is a wish for the agent, but that can be overlooked in favour of properties in higher layers.

In the sequel, we will refine the practical frameworks of definition 13 to allow for reasoning about goals, taking into account preferences.

Definition 16. Given $\mathcal{L}_{a g}$, a (full) practical framework is a tuple $\langle\epsilon, \mathcal{D}, \mathcal{G}, D, G\rangle$ where

- $\epsilon, \mathcal{D}, \mathcal{G}$ and $D$ are as in definition 13 and

- $G$ is a set of preference rules wrt $\mathcal{G}$ and some naming (and wrt $\mathcal{L}_{a g}$ ).

In the remainder of the paper, we will assume that the naming is the identity function, associating $g$ to each goal $g \in \mathcal{G}$. Moreover, for simplicity, until section 7 , we will ignore preferences over decisions and assume that $D=\{\}$. The translation of definition 14 can be generalised so that rules in $G$ are treated in a similar manner as preference rules in $\epsilon$ as in definition 12. This is illustrated by the following example.

Example 5. Consider $\pi=\langle\epsilon, \mathcal{D}, \mathcal{G},\{\}, G\rangle$ where $\epsilon=\left\{d_{1} \rightarrow p ; d_{2} \rightarrow \neg p\right\}, \mathcal{D}=\left\{d_{1}, d_{2}\right\}$, $\mathcal{G}=\{p, \neg p\}$ and $G=\{u \rightarrow p \succ \neg p ; v \rightarrow \neg p \succ p\}$, where $\mathcal{L}_{a g}=\left\{p, \neg p, u, v, d_{1}, d_{2}\right\}$. For example, in the context of the business migration scenario of section 2, $p$ may represent ease of accessibility by land, guaranteed for location $d_{1}\left(d_{1} \rightarrow p\right)$ but not true for location $d_{2}\left(d_{2} \rightarrow \neg p\right)$. The investor would prefer $p$ or $\neg p$ depending on whether it will transport goods by air $(u)$ or by land $(v)$, respectively.

Then, in $\left\langle\mathcal{L}_{\pi}, \mathcal{R}_{\pi}, \mathcal{A}_{\pi},-\right\rangle, \mathcal{R}_{\pi}$ is

$$
\begin{aligned}
& p \leftarrow b_{1} ; \quad b_{1} \leftarrow d_{1}, a_{1} ; \quad c_{1} \leftarrow \neg p \succ p ; \quad c_{1} \leftarrow b_{2} \\
& \neg p \leftarrow b_{2} ; \quad b_{2} \leftarrow d_{2}, a_{2} ; \quad c_{2} \leftarrow p \succ \neg p ; \quad c_{2} \leftarrow b_{1} \\
& p \succ \neg p \leftarrow b_{3} ; \quad b_{3} \leftarrow u, a_{3} ; \quad c_{3} \leftarrow b_{4} ; \\
& \neg p \succ p \leftarrow b_{4} \quad b_{4} \leftarrow v, a_{4} ; \quad c_{4} \leftarrow b_{3} ;
\end{aligned}
$$


where $\mathcal{A}_{\pi}=\left\{a_{1}, \ldots, a_{4}, d_{1}, d_{2}\right\}$ and $\overline{a_{i}}=c_{i}$, for all $i=1, \ldots, 4$. The translation prevents goals for being achieved (by making the appropriate decisions) when more preferred goals can also be achieved. It also prevents, in case no preferences can be applied, that conflicting goals can be achieved. In the example, if $u$ is an additional fact in $\epsilon$, then $p$ is the only goal to be pursued, and decision $d_{1}$ needs to be made, according to any argumentation semantics for $\left\langle\mathcal{L}_{\pi}, \mathcal{R}_{\pi}, \mathcal{A}_{\pi},-\right\rangle$.

So far we have assumed that the set of goals $\mathcal{G}$ is fixed. However, in general agents may need to adopt goals dynamically, e.g. as a reaction to observations that the agent makes in its environment. For example, in the case of business relocation, a financial crisis may force the agent to reconsider its investment goals. This could be accommodated, e.g. following [13], by replacing $\mathcal{G}$ with a set of defeasible rules (whose conclusions would be potential goals) with preferences, allowing to determine the most important goals for the agent to adopt at any given time.

\section{Conclusions}

We have shown how assumption-based argumentation can support reasoning with defeasible (uncertain) conflicting beliefs, alternative decisions, and possibly incompatible goals, all ranked using dynamic, qualitative preferences. This reasoning is core for service selection and composition in general, and in the motivating scenarios (business migration and earth observation applications) in particular.

The problem we have analysed is an example of argumentation for practical reasoning, trying to answer questions such as: "how to achieve a given purpose best, given some circumstances?" [2]. Since the value of the required goals cannot be evaluated with certainty, conventional multi-issue decision making techniques cannot be directly applied. Our approach realies upon (various mappings onto) assumption-based argumentation. As a by-product of these mappings, the computational techniques [8-10,12] for assumption-based argumentation can be directly deployed to provide a computational counterpart of our approach and realise argumentative agents for service selection and composition.

We have focused on the decision-making for selection and composition of services given some information (beliefs) available to the agents. Instead, within the larger ARGUGRID picture and in other work (e.g. $[16,15])$, agents communicate with one another in order to exchange/evaluate information acquired dynamically. In particular, it would be interesting to study the possibility for arguments to be exchanged amongst agents and with the users, as justifications for the agents' decisions. The communicatin with the user would be important, e.g. for business migration where it is important that the investor is presented with an appropriate analysis of the advantages and disadvantages of potential choices/decisions.

Our work also has a number of other limitations. First, we see decisions as full plans. However, they could be high-level decisions, which could in turn 
be definable in terms of atomic decisions, namely the actions composing the plans, in a hierachical fashion. Moreover, we ignore the possibility that in general actions may be definiable in terms of other actions, as in "counts-as" rules. Furthermore, we have not studied the interaction between preferences of goals and decisions: e.g., how to deal with cases where one goal is preferred to another, but the decision to achieve the first is less preferred than the one to achieve the second?

There are a number of approaches for reasoning about beliefs and goals, we will focus our comparison here on approaches using argumentation. For example, [1] uses argumentation in a similar context as the one we have considered here, but considering stratification over goals and beliefs. [2] also sees decisions as assumptions, but uses an accrual mechanism to determine the best decision. To the best of our knowledge, no existing approach considers dynamic preferences for beliefs, decisions, goals, of the type we have considered here, although [13] does consider dynamic preferences over goals. Finally, some work exists in dealing with service composition in an agent-oriented setting. For example, [15] proposes to use games for service composition, focusing on interactions amongst agents and in particular evaluating their competence to provide services.

\section{Acknowledgements}

The author has been supported by a UK Royal Academy of Engineering/ Leverhulme Trust senior fellowship and by the Sixth Framework IST programme of the EC, under the 035200 ARGUGRID project.

\section{References}

1. L. Amgoud. A unified setting for inference and decision: an argumentation-based approach. In Proc. IJCAI Workshop on Computational Models of Natural Arguments, 2005.

2. T. Bench-Capon and H. Prakken. Justifying actions by accruing arguments. In 1st International Conference on Computational Models of Argument (COMMA), 2006.

3. A. Bondarenko, P. Dung, R. Kowalski, and F. Toni. An abstract, argumentationtheoretic framework for default reasoning. Artificial Intelligence, 93(1-2):63-101, 1997.

4. G. Brewka. Well-founded semantics for extended logic programs with dynamic preferences. Journal of Artificial Intelligence Research, 4:19, 1996.

5. M. Caminada and L. Amgoud. An axiomatic account of formal argumentation. In Proc. AAAI, 2005.

6. Y. Dimopoulos, B. Nebel, and F. Toni. On the computational complexity of assumption-based argumentation for default reasoning. Artificial Intelligence, 141:57-78, 2002.

7. P. Dung. The acceptability of arguments and its fundamental role in non-monotonic reasoning and logic programming and n-person game. Artificial Intelligence, 77:321-357, 1995. 
8. P. Dung, R. Kowalski, and F. Toni. Dialectic proof procedures for assumptionbased, admissible argumentation. Artificial Intelligence, 170:114-159, 2006.

9. P. Dung, P. Mancarella, and F. Toni. A dialectic procedure for sceptical, assumption-based argumentation. In 1st International Conference on Computational Models of Argument (COMMA), 2006.

10. P. Dung, P. Mancarella, and F. Toni. Computing ideal sceptical argumentation. Artificial Intelligence - Special Issue on Argumentation in Artificial Intelligence, 171(10-15):642-674, July-October 2007.

11. I. Foster, N. Jennings, and C. Kesselman. Brain meets brawn: why grid and agents need each other. In Proc. AAMAS, 2004.

12. D. Gaertner and F. Toni. A credulous and sceptical argumentation system. In Proc. of ArgNMR, 2007.

13. A. C. Kakas and P. Moraitis. Argumentation based decision making for autonomous agents. In Proc. AAMAS, pages 883-890, 2003.

14. R. A. Kowalski and F. Toni. Abstract argumentation. Journal of Artificial Intelligence and Law, Special Issue on Logical Models of Argumentation, 4(3-4):275-296, 1996.

15. K.Stathis, G.K.Lekeas, and C. Kloukinas. Competence checking for the global e-service society using games. In Engineering Societies in the Agents World (ESAW06). Springer, 2007.

16. M. Morge. A dialectics multiagent system in which argumentative agents play and arbitrate to reach an agreement. In Proc.1st Workshop on Argumentation in Artificial Intelligence and Law, 2005.

17. D. Nute. Defeasible reasoning. In J. H. Fetzer, editor, Aspects of Artificial Intelligence, pages 251-288. Kluwer Academic Publishers, 1987.

18. H. Prakken and G. Sartor. Argument-based extended logic programming with defeasible priorities. Journal of Applied Non-Classical Logics, 7(1):25-75, 1997.

19. T. Stournaras, D. Dimitrelos, A. Tabasco, J. Barba, D. Pedrazzani, M. Yage, T. An, P. Dung, N. Hung, V. D. Khoi, and P. M. Thang. e-business application scenarios. In T. Stournaras, editor, ARGUGRID deliverable D.1.2, 2007.

20. F. Toni. Assumption-based argumentation for closed and consistent defeasible reasoning. In Proc. JURISIN 2007, in association with The 21th Annual Conference of The Japanese Society for Artificial Intelligence (JSAI2007), 2007. 